EPJ manuscript No.

(will be inserted by the editor)

\title{
Similar final states from different initial states using new exact solutions of relativistic hydrodynamics
}

\author{
M. Csanád ${ }^{1, a}$, M. I. Nagy ${ }^{1, b}$, and T. Csörgö ${ }^{2, c}$ \\ 1 ELTE, Eötvös Loránd University, H - 1117 Budapest, Pázmány P. s. 1/A, Hungary \\ 2 MTA KFKI RMKI, H-1525 Budapest 114, POBox 49, Hungary
}

\begin{abstract}
We present exact, analytic and simple solutions of relativistic perfect fluid hydrodynamics. The solutions allow us to calculate the rapidity distribution of the particles produced at the freeze-out, and fit them to the measured rapidity distribution data. We also give an advanced estimation of the energy density reached in heavy ion collisions, and an improved estimation of the life-time of the reaction.
\end{abstract}

\section{Introduction}

Since the birth of hydrodynamics, there were many interesting exact solutions found to the complicated non-linear coupled differential equations of them. In contemporary research, such as the description of collective properties of high-energy elementary particle and heavy ion reactions, one has to deal with not only hydrodynamics but relativistic hydrodynamics. This is an even more complicated topic, and likely this is the reason why only a few exact solutions exist for relativistic hydrodynamics, in contrast to the nonrelativistic case.

Exact solutions are important in at least two ways: first, they can be used to test numerical codes reliably. Second, they provide an invaluable insight into the details of the evolution of the matter created in high-energy reactions. After recalling some presently known solutions, such as the Landau-Khalatnikov solution and the Hwa-Bjorken solution, we present a class of accelerating exact and explicit solutions of relativistic hydrodynamics. These solutions are advantageous compared to the previously mentioned ones. Then we show how the new solutions can be applied to describe the evolution of the matter created in high energy reactions. Our treatment uses simple formulas and takes the presence of acceleration into account.

This article is based on refs. 11213. These results are extended here with more detailed simulations that indicate that even for a fixed equation of state, different initial conditions could lead to the same freeze-out distributions at mid-rapidity. However, the width of the rapidity distribution can be utilized to select from among these time evolution scenarios.

\section{Basic equations}

In this section we recapitulate the equations of relativistic hydrodynamics. We will use the following notations: $x^{\mu}=(t, \mathbf{r})$ is the coordinate four-vector, $\mathbf{r}=\left(r_{x}, r_{y}, r_{z}\right)$ is the coordinate three-vector. The metric tensor is $g_{\mu \nu}=\operatorname{diag}(1,-1,-1,-1)$, the four-velocity field is $u^{\mu}$, the normalization is $u^{\mu} u_{\mu}=1$. The three-velocity $\mathbf{v}$ is defined as $u^{\mu}=\gamma(1, \mathbf{v})$, with $\gamma=$

\footnotetext{
a e-mail: csanad@elte.hu

b e-mail: nagymarci@rmki.kfki.hu

c e-mail: csorgo@sunserv.kfki.hu
} 
$\left(1-v^{2}\right)^{-1 / 2}$. We denote the so-called comoving derivative by $\frac{\mathrm{d}}{\mathrm{d} t}$, that is, $\frac{\mathrm{d}}{\mathrm{d} t}=\frac{\partial}{\partial t}+\mathbf{v} \nabla$. The thermodynamical quantities are the following: $\varepsilon$ is the energy density, $p$ is the pressure, $w=\varepsilon+p$ is the enthalpy density, $T$ is the temperature, $\sigma$ is the entropy density. When there are some (conserved or non-conserved) charges present, we denote them by $n_{i}$, and the corresponding chemical potentials by $\mu_{i}$.

The equations of relativistic hydrodynamics are obtained in the simplest way by Landau's heuristic argumentation: for perfect fluid (without viscosity and heat conductivity) the energymomentum tensor is

$$
T_{\mu \nu}=\operatorname{diag}(\varepsilon, p, p, p)
$$

in the local rest frame, so we have

$$
T_{\mu \nu}=w u_{\mu} u_{\nu}-p g_{\mu \nu}
$$

in arbitrary frame. Projecting the energy-momentum conservation law $\partial_{\nu} T^{\mu \nu}=0$ orthogonal and parallel to $u^{\mu}$, we obtain the relativistic Euler equation and the energy conservation equation as

$$
\begin{aligned}
w u^{\nu} \partial_{\nu} u^{\mu} & =\left(g^{\mu \rho}-u^{\mu} u^{\rho}\right) \partial_{\rho} p \\
w \partial_{\mu} u^{\mu} & =-u^{\mu} \partial_{\mu} \varepsilon
\end{aligned}
$$

The charge conservation equation (for one charge) is

$$
\partial_{\mu}\left(n u^{\mu}\right)=0
$$

but if there are many different particles with the same charge, then this has to be supplemented by the chemical potentials. For instance, in case of baryonic or electric charge, particles and antiparticles carry opposite charges, and they chemical potentials are the same but of opposite sign, so we have

$$
\sum_{i} \mu_{i} \partial_{\mu}\left(n_{i} u^{\mu}\right)=0
$$

\section{Known solutions}

In this section we summarize some already known exact results: the renowned Landau-Khalatnikov solution, the Hwa-Bjorken solution and other recent multi-dimensional solutions.

\subsection{The Landau-Khalatnikov solution}

Landau invented relativistic hydrodynamics in the early 1950s and proposed the application of it to describe high-energy particle reactions (in that time mostly cosmic ray events). In a collaboration with I. M. Khalatnikov, they found the first exact solution [4]5, which is a 1+1 dimensional implicit solution, with $\varepsilon=3 p$ equation of state (EoS). The Landau-Khalatnikov solution has nice realistic features: it is an accelerating one, the initial condition describes a finite piece of matter at rest, then it starts to expand. Another important feature is that the rapidity distribution of the particles is approximately Gaussian. However, the solution itself is very complicated, since the independent variables - the time and spatial coordinate - are given in terms of horrendous integral formulas involving Bessel functions integrated over the temperature and the fluid rapidity "variables". Thus we don't quote the whole result here. 


\begin{tabular}{|c|c|c|c|c|}
\hline Case & $\lambda$ & $d$ & $\kappa$ & $\phi_{\lambda}$ \\
\hline a.) & 1 & $\in \mathbb{R}$ & $\in \mathbb{R}$ & 0 \\
b.) & 2 & $\in \mathbb{R}$ & $d$ & 0 \\
c.) & $\frac{1}{2}$ & $\in \mathbb{R}$ & 1 & $\frac{\kappa+1}{\kappa}$ \\
d.) & $\frac{3}{2}$ & $\in \mathbb{R}$ & $\frac{4 d-1}{3}$ & $\frac{\kappa+1}{\kappa}$ \\
e.) & $\in \mathbb{R}$ & 1 & 1 & 0 \\
\hline
\end{tabular}

Table 1. The new family of solutions.

\subsection{The Hwa-Bjorken solution}

The Hwa-Bjorken solution [6 7] is a $1+1$ dimensional, expanding accelerationless solution. It is simple and explicit, this is its main advantage compared to the Landau-Khalatnikov solution. It is written down in the easiest way in Rindler-coordinates $\tau$ and $\eta$, which are defined by

$$
t=\tau \cosh \eta \quad, \quad r=\tau \sinh \eta
$$

with $r$ being the spatial coordinate. The Hwa-Bjorken solution is given by the velocity and entropy profiles

$$
v=\tanh \eta=\frac{r}{t} \quad, \quad \sigma=\sigma_{0} \frac{\tau_{0}}{\tau},
$$

where the subscript ${ }_{0}$ refers to the initial condition. It works for $\epsilon+B=\kappa(p+B)$, for arbitrary values of $\kappa$ and $B$. This boost-invariant solution results in a constant rapidity distribution of the particles. This is a rough prediction clearly not valid at the present experimental situations. However, Bjorken has given a simple estimate of the initial energy density of the reaction based on this solution, this is why it became so renowned. In the next section we present such solutions that are simple and explicit (as the Hwa-Bjorken solution) and are accelerating, and yield realistic rapidity distributions (as the Landau-Khalatnikov solution).

\section{New simple analytic solutions}

The Landau-Khalatnikov solution contains acceleration, and yields a finite, realistic, Gaussianlike rapidity distribution. On the other hand, the Hwa-Bjorken solution is very simple and easy to handle, this is why it became so important, although the accelerationless, boost-invariant flow profile and the constant rapidity distribution obviously does not agree with the results of real high-energy experiments.

We found such new, analytic, explicit and simple solutions, which do not lack acceleration, and yield finite, realistic rapidity distributions 12 . We found solutions for $1+1$ dimensions, and also spherical flows in arbitrary number of spatial dimensions ( $d$ will denote their number). For presenting the solutions we use Rindler-coordinates, which are defined by eq. (7) for $t>r$, that is ,,inside the lightcone". (From now on $t$ is the time and $r$ is the radial coordinate in the $1+\mathrm{d}$ dimensional case, and the single spatial coordinate in the $1+1$ dimensional case.)

For a discussion of the mathematical derivations in greater detail, apart from Refs. 112], we recommend Ref. 3. Here we present only the main results. The velocity and the pressure is given by

$$
v=\tanh (\lambda \eta) \quad, \quad p=p_{0}\left(\frac{\tau_{0}}{\tau}\right)^{\lambda d \frac{\kappa+1}{\kappa}} \frac{1}{\cosh ^{\phi_{\lambda}(d-1)} \frac{\eta}{2}} .
$$

The value of the constants $\lambda, \phi_{\lambda}, d$ and $\kappa$ are constrained: different set of values yield different solutions. Table 1 shows the possible cases, every row of the table being a solution. In the following we discuss them separately.

- Case a) isn't a new solution, it is just the well-known Hwa-Bjorken solution in 1+1 dimensions, and the only recently discovered Buda-Lund type of solutions [12 13] in $1+\mathrm{d}$ dimensions. We quoted it for the sake of completeness, since this solution is also a member of the class. Apart from this case, all the other solutions possess non-vanishing acceleration. 
- Case b) was found first, with other methods. It describes spherical expansion in $d$ dimensions. The EoS is constrained in a way that $\kappa$ must be equal to $d$. (For instance, in case of threedimensional expansion $\kappa=3$, which is the EoS of an ultra-relativistic ideal photon gas.) It is interesting to calculate the equation of trajectories $R(t)$ from the velocity field in Minkowskian coordinates:

$$
v=\frac{2 t r}{r^{2}+t^{2}} \quad \Rightarrow \quad R(t)=\frac{1}{a_{0}}\left(\sqrt{1+\left(a_{0} t\right)^{2}}+1\right)
$$

with $a_{0}=\frac{2 r_{0}}{\left|r_{0}^{2}-t_{0}^{2}\right|}$. So the fluid elements have constant $a_{0}$ acceleration in the local rest frame.

- Case $c$ ) and $d$ ) were found first by T. S. Biró in $1+3$ dimensions [14, we generalized them to arbitrary number of spatial dimensions. These solutions are also accelerating, and since $\phi_{\lambda} \neq 0$, if $d \neq 1$, the pressure field explicitly depends on $\eta$, so the solution is finite in $\eta$.

- Case $e$ ) has a remarkably general velocity field: the $\lambda$ parameter can be arbitrary. We call $\lambda$ acceleration parameter, because it somehow governs the acceleration of the flow. On the other hand, this solution works only for $d=1$ and $\kappa=1$, which is obviously a drawback. Nevertheless, this solution can be considered as a ,smooth extrapolation" between the previous cases. In the next section we shall use this solution to calculate the rapidity distribution, and see that the width of it is controlled by the value of $\lambda$. Hence in principle, the value of the parameter $\lambda$ of the hydrodynamical solution can be obtained from measurements.

For a more thorough review of these solutions we recommend ref. [3].

For illustration, in Fig. 1 we plot the spatial temperature distribution at various points of the evolution of a collision for different $\lambda$ values. All the plotted solutions go to the same final temperature at mid-rapidity $(\eta=0)$, although their initial temperature and acceleration parameter $\lambda$ are different. We shall see in the next section, that solutions with different acceleration parameter $\lambda$ have different rapidity distributions, and the acceleration parameter can be determined from the measurement of the widths of the rapidity distribution.

\section{Rapidity distribution}

We calculated the rapidity distribution, $\frac{\mathrm{d} n}{\mathrm{~d} y}$ of the particles numerically and (approximately) analytically as well. We used the solutions discussed in the previous section as case $e$.), that is, where the $\lambda$ parameter is arbitrary. We assumed that at a certain freeze-out massive particles appear. (In the figures of this paper we used pions with $m=140 \mathrm{MeV}$, but other particles can be used as well - and their contribution can be added to the one of pions). We have chosen the freeze-out condition as follows: the temperature in $\eta=0$ should reach a given $T_{f}$ value (subscript $f$ means freeze-out) and the freeze-out hypersurface should be pseudo-orthogonal to the four-velocity field $u_{\mu}(x)$. The equation of this hypersurface is

$$
\left(\frac{\tau_{f}}{\tau}\right)^{\lambda-1} \cosh ((\lambda-1) \eta)=1
$$

The details of the calculation of $\frac{\mathrm{d} n}{\mathrm{~d} y}$ is found in refs. [13]. We only quote the result here: using a saddle-point integration in $\eta$, for $\lambda>1 / 2, m / T_{f} \gg 1$ and $\nu_{\sigma}(s)=1$ we got

$$
\left.\frac{\mathrm{d} n}{\mathrm{~d} y} \approx \frac{\mathrm{d} n}{\mathrm{~d} y}\right|_{y=0} \cosh ^{-\frac{\alpha}{2}-1}\left(\frac{y}{\alpha}\right) e^{-\frac{m}{T_{f}}\left[\cosh ^{\alpha}\left(\frac{y}{\alpha}\right)-1\right]}
$$

where $\alpha=\frac{2 \lambda-1}{\lambda-1}$. Some typical cases are plotted in Fig. 2. This figure is just an illustration, the parameter values on this figure are not realistic ones. 

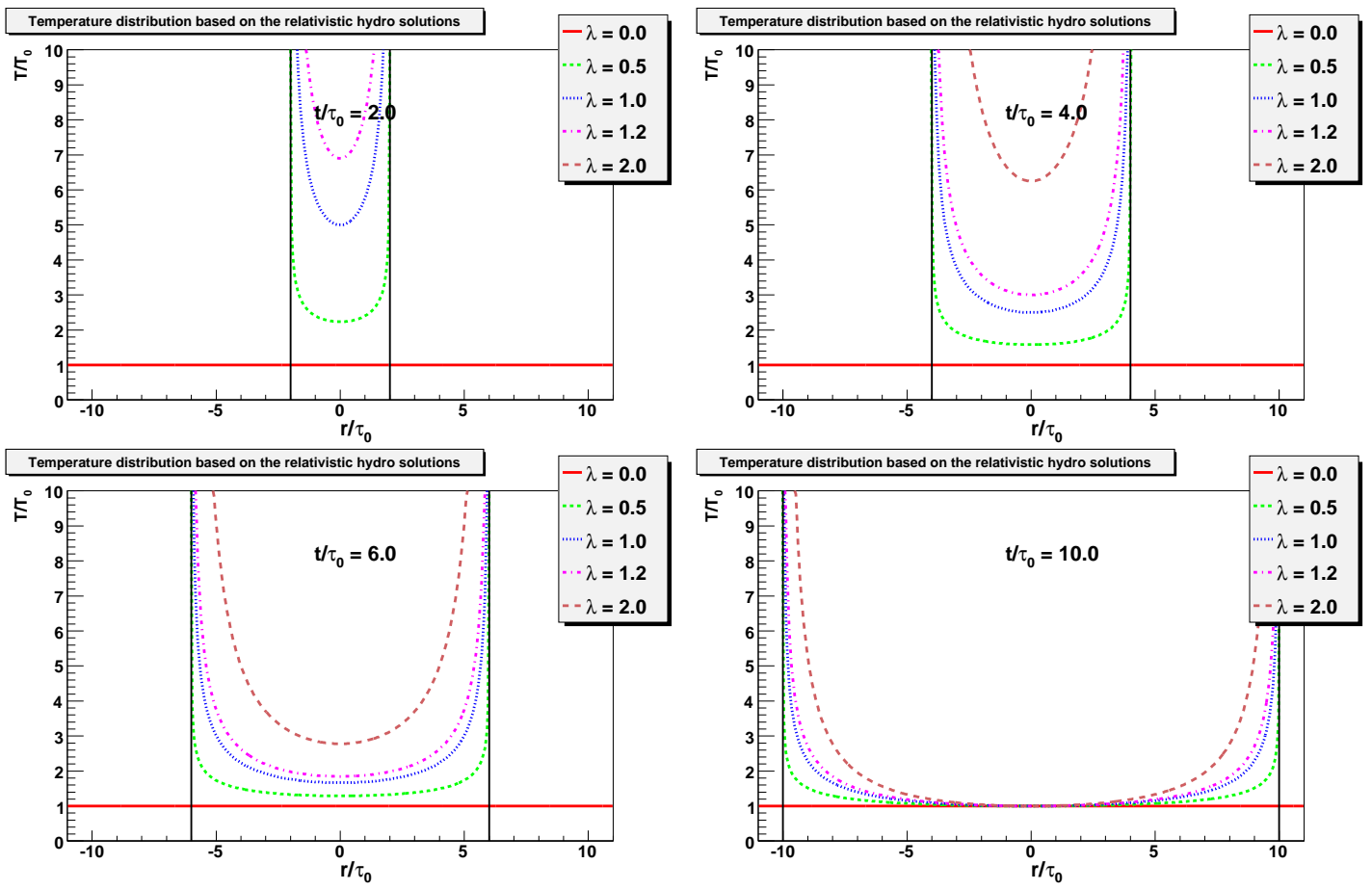

Fig. 1. (Color online) These plots show the temperature distribution of different one dimensional solutions (case e. of Table 1 with different $\lambda$ parameters) with different initial temperature. All the plotted solutions go to the same final temperature at mid-rapidity $(\eta=0)$. The $\lambda=1$ solution corresponds to the Hwa-Bjorken solution. All the $\lambda>0$ solutions are shown within the light-cone (vertical solid black lines). The horizontal solid red line indicates the $\lambda=0$ case, corresponding to a static, infinite, homogeneous medium with a constant temperature, and its domain extends beyond the light-cone, while for $\lambda>0$ we plot the solutions at $r<t$ only.

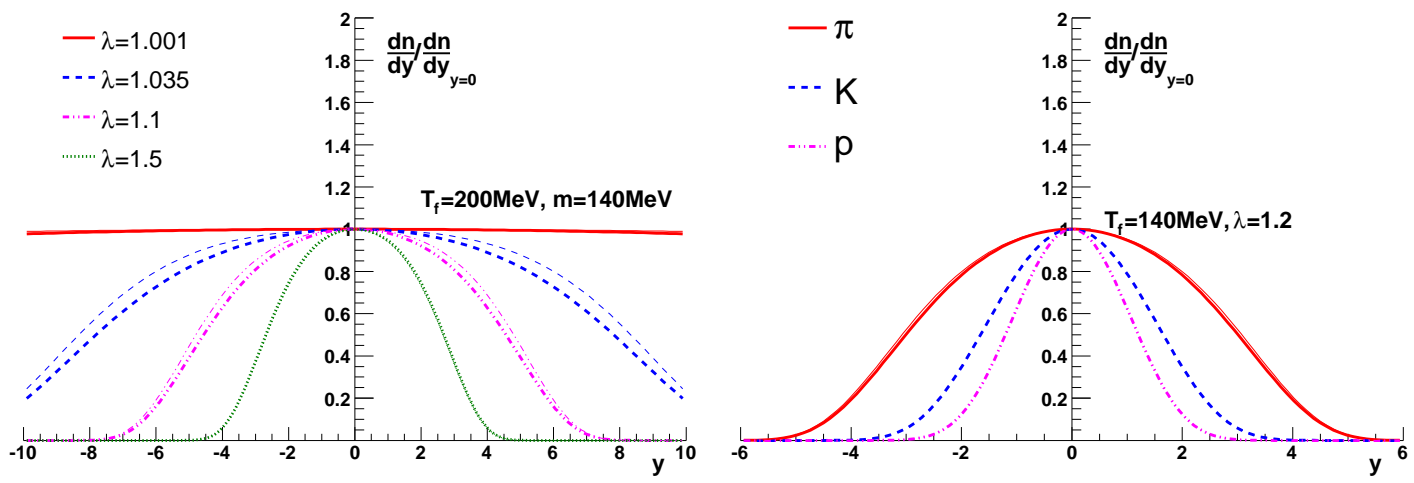

Fig. 2. (Color online) Normalized rapidity distributions from the new solutions in $1+1$ dimensions case e.) of Table1- for various $\lambda, T_{f}$ and $m$ values. Thick lines show the result of numerical integration, thin lines the analytic approximation from eq. (12). For $\lambda>1$ and not too big $T_{f}$ it can be used within about $10 \%$ error.

\section{Energy density estimation}

Now as we have an analytic approximation for the rapidity distribution, we are able to fit it to real experimental data and extract the $\lambda$ acceleration parameter from them. Since our treatment includes acceleration effects, the new solutions provide a more realistic picture of the initial period of high energy reactions. In this section we show how this can be used for improving the famous energy density estimation made by Bjorken. It is clear that initial energy 


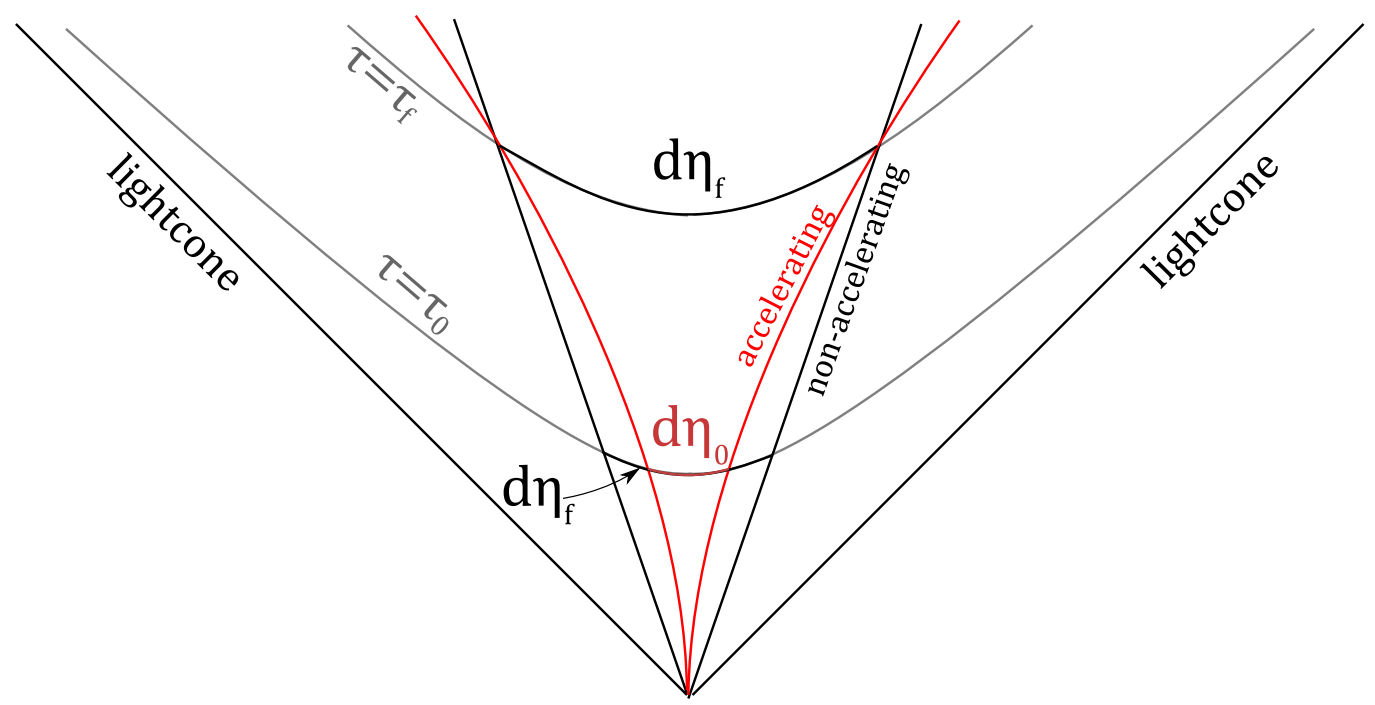

Fig. 3. (Color online) This figure shows that if there is no acceleration, $\eta_{0}=\eta_{f}$, but for the accelerating case, a correction factor has to be applied.

density is a quantity of crucial importance when one wants to interpret the conclusions drawn from high energy experiments.

We follow Bjorken's method 7 and modify it when acceleration effects become important. Let us focus on a thin transverse piece of the produced matter at mid-rapidity, as seen on by Fig. 2 of ref. [7. The radius $R$ of this slab is estimated by the radius of the colliding hadrons or nuclei: $R=1.18 A^{1 / 3} \mathrm{fm}$. The volume is $d V=\left(R^{2} \pi\right) \tau \mathrm{d} \eta$, where $\tau$ is the proper time of observation and $\mathrm{d} \eta$ is the space-time rapidity extent of the slab. The energy content $d E=\left\langle m_{t}\right\rangle d n$, where $\left\langle m_{t}\right\rangle$ is the average transverse mass at mid-rapidity, so similarly to Bjorken, the initial energy density is

$$
\varepsilon_{0}=\frac{\left\langle m_{t}\right\rangle}{\left(R^{2} \pi\right) \tau_{0}} \frac{d n}{d \eta_{0}}
$$

Here $\tau_{0}$ is the proper-time of thermalization, estimated by Bjorken as $\tau_{0} \approx 1 \mathrm{fm}$. For accelerationless, boost-invariant Hwa-Bjorken flows $\eta_{0}=\eta_{f}=y$, however, for accelerating solutions one has to apply a correction of

$$
\frac{\partial y}{\partial \eta_{f}} \frac{\partial \eta_{f}}{\partial \eta_{0}}=(2 \lambda-1)\left(\tau_{f} / \tau_{0}\right)^{\lambda-1}
$$

(see Fig. 31). These two factors contain the acceleration effects on the energy density estimation, see ref. [3] for details.

So the initial energy density $\varepsilon_{0}$ can be accessed by an advanced estimation $\varepsilon_{c}$ as

$$
\frac{\varepsilon_{c}}{\varepsilon_{B j}}=(2 \lambda-1)\left(\frac{\tau_{f}}{\tau_{0}}\right)^{\lambda-1} \quad, \quad \varepsilon_{B j}=\frac{\left\langle m_{t}\right\rangle}{\left(R^{2} \pi\right) \tau_{0}} \frac{d n}{d y} .
$$

Here $\varepsilon_{B j}$ is the Bjorken estimation, which is recovered if $\frac{\mathrm{d} n}{\mathrm{~d} y}$ is flat (i.e. $\lambda=1$ ), but if $\lambda>1$, i.e. the flow is accelerating, both correction factors are greater than 1 , so $\varepsilon_{0}$ is under-estimated by the Bjorken formula. Fig. 4 shows our fits to BRAHMS $d n / d y$ data [15]. From these fits we have found $\lambda=1.18 \pm 0.01$.

Using the Bjorken estimate of $\varepsilon_{B j}=5 \mathrm{GeV} / \mathrm{fm}^{3}$ as given in ref. [16], and $\tau_{f} / \tau_{0}=8 \pm 2$ $\mathrm{fm} / \mathrm{c}$, we find an initial energy density of $\varepsilon_{c}=(2.0 \pm 0.1) \varepsilon_{B j}=10.0 \pm 0.5 \mathrm{GeV} / \mathrm{fm}^{3}$. 

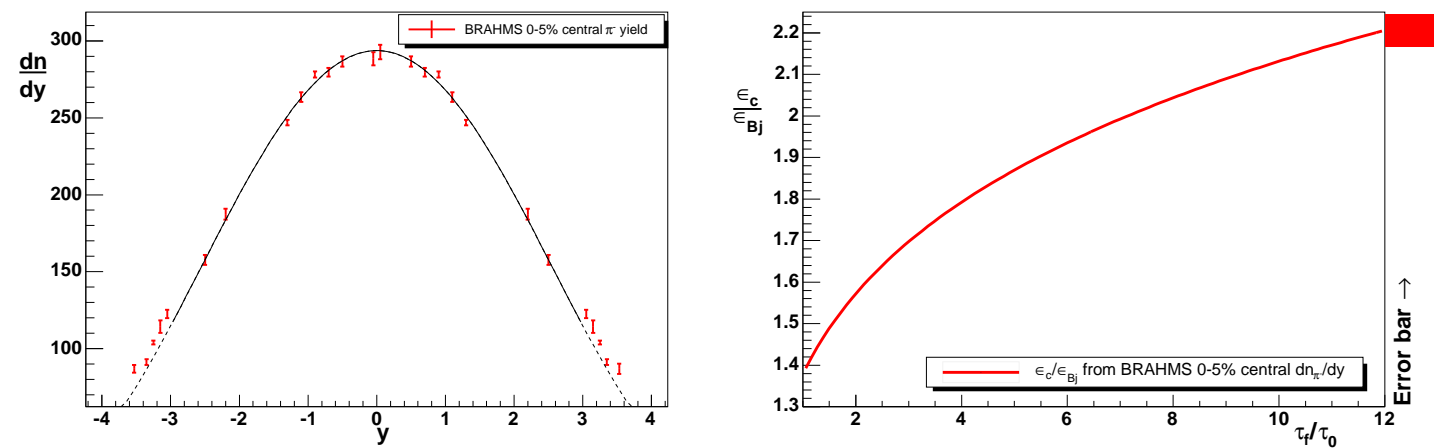

Fig. 4. (Color online) Left panel: $d n / d y$ data of negative pions, as measured by the BRAHMS collaboration [15] in central (0-5\%) Au+Au collisions at $\sqrt{s_{N N}}=200 \mathrm{GeV}$, fitted with eq. (12) $(1+3$ dimensional case). The fit range was $-3<y<3$, to exclude target and projectile rapidity region, CL $=0.6 \%$. Right panel: $\varepsilon_{c} / \varepsilon_{B j}$ ratio as a function of $\tau_{f} / \tau_{0}$.

\section{Life-time estimation}

As another application, let us focus on the life-time of a high energy reaction. For a Hwa-Bjorken type of accelerationless, coasting longitudinal flow, Sinyukov and Makhlin [17] determined the longitudinal length of homogeneity as

$$
R_{l o n g}=\sqrt{\frac{T_{f}}{m_{t}}} \tau_{B j}
$$

Here $m_{t}$ is the transverse mass and $\tau_{B j}$ is the (Bjorken) freeze-out time. This result provides a means to determine the life-time of the reaction, if one simply identifies it with $\tau_{B j}$. However, if the flow is accelerating, the case is a little bit more complicated: the estimated origin of the trajectories is shifted back in proper-time, so the life-time of the reaction is under-estimated by $\tau_{B j}$. From our solutions we have (for a broad but finite rapidity distribution, so that the saddle-point approximation can be used):

$$
R_{\text {long }}=\sqrt{\frac{T_{f}}{m_{t}}} \frac{\tau_{c}}{\lambda} \Rightarrow \tau_{c}=\lambda \tau_{B j}
$$

Thus the new estimation of the life-time, $\tau_{c}$ contains a $\lambda$ multiplication factor. BRAHMS rapidity distributions in Fig. 4 yield $\lambda=1.18 \pm 0.01$, so they imply a $18 \pm 1 \%$ increase in the estimated life-time of the reaction.

\section{Summary}

We presented a class of simple solutions of relativistic perfect fluid hydrodynamics. Because the new solutions are accelerating, but still explicit and exact, they can be utilized in the description of high energy reactions in a more reliable way than either the Hwa-Bjorken of the Landau-Khalatnikov solution. We have shown, that even for a fixed equation of state, different initial conditions could lead to the same freeze-out distributions at mid-rapidity. However, these solutions of different acceleration parameter $\lambda$ have different width of their rapidity distributions, and the acceleration parameter can thus be determined from the measurement of the widths of the rapidity distribution. We have calculated the rapidity distribution of the produced particles, and showed two possible applications: and advanced estimation of the initial energy density of the high energy reaction and an advanced life-time estimation. We fitted the analytic approximation of the rapidity distribution to experimental data (measured by the BRAHMS collaboration at RHIC) and determined the $\lambda$ acceleration parameter of the flow. From it we 
concluded that the energy density estimations based on Bjorken's method has to be corrected by a factor of 2 . We also obtained a correction to the life-time measurements: a $\sim 20 \%$ increase is due to the presence of acceleration in the initial evolution of the matter. Thus the effects of work, done even by the fluid cell at mid-rapidity, can be determined from the measurement of the widht of the rapidity distribution and this information is important for an advanced estimation of initial energy densities and the life-time of the rapidity distribution.

\section{References}

1. T. Csörgő, M. I. Nagy, and M. Csanád, nucl-th/0605070

2. T. Csörgö, M. I. Nagy, and M. Csanád, nucl-th/0702043

3. M. I. Nagy, T. Csörgő and M. Csanád, arXiv:0709.3677 [nucl-th].

4. L. D. Landau, Izv. Akad. Nauk SSSR Ser. Fiz. 17, 51 (1953).

5. I. M. Khalatnikov, Zhur. Eksp. Teor. Fiz. 27, 529 (1954).

6. R. C. Hwa, Phys. Rev. D10, 2260 (1974).

7. J. D. Bjorken, Phys. Rev. D27, 140 (1983).

8. Y. M. Sinyukov and I. A. Karpenko, Acta Phys. Hung. A25, 141 (2006).

9. Y. M. Sinyukov and I. A. Karpenko, nucl-th/0505041

10. T. S. Biró, Phys. Lett. B487, 133 (2000).

11. T. S. Biró, Phys. Lett. B474, 21 (2000).

12. T. Csörgő, F. Grassi, Y. Hama and T. Kodama, Phys. Lett. B 565, 107 (2003)

13. T. Csörgö, L. P. Csernai, Y. Hama and T. Kodama, Heavy Ion Phys. A 21, 73 (2004)

14. T. S. Biró, private communication. The authors are grateful to him for this comment.

15. I. G. Bearden et al. [BRAHMS Collaboration], Phys. Rev. Lett. 94, 162301 (2005)

16. I. Arsene et al. [BRAHMS Collaboration], Nucl. Phys. A 757, 1 (2005)

17. A. Makhlin \& Yu. Sinyukov, Z. Phys. C39, 69 (1988). 